\title{
Soluble Vascular Cell Adhesion Molecule and Cardiovascular Disease: An Epidemiological View
}

\author{
Kazumasa Yamagishi ${ }^{1}$ and Hiroyasu Iso $^{2}$ \\ ${ }^{1}$ Department of Public Health Medicine, Faculty of Medicine, University of Tsukuba, Tsukuba, Japan \\ ${ }^{2}$ Public Health, Department of Social Medicine, Osaka University Graduate School of Medicine, Suita, Japan
}

\section{See article vol. 24: 804-818}

Novel biomarkers for primary prevention of cardiovascular disease have been evaluated worldwide, but few appeared to improve risk prediction beyond classical risk factors, such as high blood pressures, dyslipidemias, and hyperglycemias ${ }^{1)}$.

Soluble cell adhesion molecules have been considered early systemic inflammation and endothelial cell activation markers ${ }^{2)}$ and risk marker for consequent development of cardiovascular disease. Thus, high levels of these circulating molecules have been hypothesized to be associated with higher risk of cardiovascular disease. Of these molecules, the evidence on the predictive role of soluble vascular cell adhesion molecule-I (VCAM-I) has been relatively limited, and most previous studies have shown no association ${ }^{3)}$.

In this issue of the Journal of Atherosclerosis and Thrombosis, Kunutsor et al. examined the association between soluble VCAM-I plasma levels and incident cardiovascular disease (coronary heart disease and stroke) in the Prevention of Renal and Vascular Endstage Disease (PREVEND) Study, a prospective cohort study of a general population living in the city of Groningen, the Netherlands ${ }^{4)}$. They found a weak correlation between VCAM-I and classical risk factors, and unexpectedly, an inverse association between VCAM-1 and risk of cardiovascular disease (primarily coronary heart disease) after adjustment for, or stratification by, classical risk factors. VCAM-I did not improve cardiovascular disease risk assessment beyond classical risk factors.

The PREVEND Study is a well-designed national

Address for correspondence: Kazumasa Yamagishi, Department of Public Health Medicine, Faculty of Medicine, University of Tsukuba, Tennodai 1-1-1, Tsukuba 305-8575, Japan

E-mail: yamagishi.kazumas.ge@u.tsukuba.ac.jp

Received: April 4, 2017

Accepted for publication: April 11, 2017 representative prospective cohort study. However, the result from a single study, when the results were not consistent with those of prior hypotheses, should be interpreted with caution. Generally, high levels of VCAM-I are considered to be linked with high risk of cardiovascular disease. The authors carefully tested the possibility of bias, such as reverse causation, confounders, and interaction with other classical factors, and noted several limitations: residual confounding, absence of repeat measurements, generalizability to other ethnicities, and plasma storage. In addition, they did not evaluate the amount of alcohol intake, and the high amount may damage endothelial function ${ }^{5}$. Further studies are needed to confirm the results, and using VCAM-1 for the prediction of future cardiovascular disease may not be practical in clinical/public health settings at this moment.

Nonetheless, this study turned the spotlight on the role of VCAM-1 on the development of cardiovascular disease. Taken together with previous studies with the null association in the general population ${ }^{3)}$, VCAM-1 may play a different role in the pathogenesis of atherosclerosis with other adhesion molecules. The authors speculated several mechanisms that may explain the difference. Biological mechanisms underlying the inverse association should be revealed to determine whether VCAM-1 could be a protective marker.

\section{Acknowledgement}

The authors thank Mr. Takahiro Itagaki for technical assistance.

\section{References}

1) Folsom AR. Classical and novel biomarkers for cardiovascular risk prediction in the United States. J Epidemiol 2013; 23: 158-162

2) Badimon L, Romero JC, Cubedo J, Borrell-Pagès M. Cir- 
culating biomarkers. Thromb Res 2012; 130: S12-S15

3) Blankenberg S, Barbaux S, Tiret L. Adhesion molecules and atherosclerosis. Atherosclerosis 2003; 170: 191-203

4) Kunutsor SK, Bakker SJL, Dullaart RPF. Soluble vascular cell adhesion molecules may be protective of future cardiovascular disease risk: Findings from the PREVEND Prospective Cohort Study. J Atheroscler Thromb 2017;
24: 804-818

5) Tanaka A, Cui R, Kitamura A, Liu K, Imano H, Yamagishi K, Kiyama M, Okada T, Iso H; CIRCS Investigators. Heavy alcohol consumption is associated with impaired endothelial function. J Atheroscler Thromb 2016; 23: $1047-1054$ 